\title{
A classification of certain submanifolds of an $S$-manifold
}

\author{
by José L. Cabrerizo, Luis M. Fernández \\ and Manuel Fernández (Sevilla)
}

\begin{abstract}
A classification theorem is obtained for submanifolds with parallel second fundamental form of an $S$-manifold whose invariant $f$-sectional curvature is constant.

0. Introduction. For manifolds with an $f$-structure, David E. Blair has introduced the analogue of the Kaehler structure in the almost complex case and the quasi-Sasakian structure in the almost contact case, defining the $S$-manifolds ([1]).

The purpose of this note is to present the following theorem about submanifolds with parallel second fundamental form of an $S$-manifold of constant invariant $f$-sectional curvature $k$ :
\end{abstract}

THEOREM 1. Let $M^{m+s}$ be a submanifold of an $S$-manifold $N^{2 n+s}(k)$ $(k \neq s)$, tangent to the structure vector fields. If the second fundamental form $\sigma$ of $M^{m+s}$ is parallel, then $M^{m+s}$ is one of the following submanifolds:

(a) an invariant submanifold of constant invariant $f$-sectional curvature $k$, immersed in $N^{2 n+s}(k)$ as a totally geodesic submanifold;

(b) an anti-invariant submanifold immersed in $\bar{M}^{2 m+s}(k)$, where $\bar{M}^{2 m+s}(k)$ is an invariant and totally geodesic submanifold of $N^{2 n+s}(k)$ of constant invariant $f$-sectional curvature $k \neq s$.

1. Preliminaries. Let $N^{n}$ be an $n$-dimensional Riemannian manifold and $M^{m}$ an $m$-dimensional submanifold of $N^{n}$. Let $g$ be the metric tensor field on $N^{n}$ as well as the induced metric on $M^{m}$. We denote by $\widetilde{\nabla}$ the covariant differentiation in $N^{n}$ and by $\nabla$ the covariant differentiation in $M^{m}$ determined by the induced metric. Let $T(N)$ (resp. $\left.T(M)\right)$ be the Lie

1985 Mathematics Subject Classification: Primary 53C40, 53C25.

Key words and phrases: S-manifolds, parallel second fundamental form.

The authors are partially supported by the project PAICYT (SPAIN) 1989. 
algebra of vector fields on $N^{n}$ (resp. on $M^{m}$ ) and $T(M)^{\perp}$ the set of all vector fields normal to $M^{m}$. The Gauss-Weingarten formulas are given by

$$
\widetilde{\nabla}_{X} Y=\nabla_{X} Y+\sigma(X, Y) \text { and } \widetilde{\nabla}_{X} V=-A_{V} X+D_{X} V,
$$

for any $X, Y \in T(M)$ and $V \in T(M)^{\perp}$, where $D$ is the connection in the normal bundle, $\sigma$ is the second fundamental form of $M^{m}$ and $A_{V}$ is the Weingarten endomorphism associated with $V . A_{V}$ and $\sigma$ are related by

$$
g\left(A_{V} X, Y\right)=g(\sigma(X, Y), V) .
$$

We denote by $\widetilde{R}$ and $R$ the curvature tensors associated with $\widetilde{\nabla}$ and $\nabla$, respectively. The Gauss equation is given by

$$
\begin{aligned}
\widetilde{R}(X, Y, Z, W)= & R(X, Y, Z, W)+g(\sigma(X, Z), \sigma(Y, W)) \\
& -g(\sigma(X, W), \sigma(Y, Z)), \quad X, Y, Z, W \in T(M) .
\end{aligned}
$$

Moreover, we have the following Codazzi equation:

$$
(\widetilde{R}(X, Y) Z)^{\perp}=\left(\nabla_{X}^{\prime} \sigma\right)(Y, Z)-\left(\nabla_{Y}^{\prime} \sigma\right)(X, Z),
$$

$X, Y, Z \in T(M)$, where $\perp$ denotes the normal projection and the covariant derivative of the second fundamental form $\sigma$ is defined as follows:

$$
\left(\nabla_{X}^{\prime} \sigma\right)(Y, Z)=D_{X} \sigma(Y, Z)-\sigma\left(\nabla_{X} Y, Z\right)-\sigma\left(Y, \nabla_{X} Z\right),
$$

$X, Y, Z \in T(M)$. The second fundamental form $\sigma$ is said to be parallel if $\nabla^{\prime} \sigma=0$.

Finally, the submanifold $M^{m}$ is said to be totally geodesic in $N^{n}$ if $\sigma \equiv 0$.

2. Submanifolds of an $S$-manifold. Let $\left(N^{2 n+s}, g\right)$ be a $(2 n+s)$ dimensional Riemannian manifold. $N^{2 n+s}$ is said to be an $S$-manifold if there exist on $N^{2 n+s}$ an $f$-structure $f([8])$ of rank $2 n$, and $s$ global vector fields $\xi_{1}, \ldots, \xi_{s}$ (structure vector fields) such that ([1]):

(i) If $\eta_{1}, \ldots, \eta_{s}$ are the dual 1 -forms of $\xi_{1}, \ldots, \xi_{s}$, then

$$
\begin{gathered}
f \xi_{\alpha}=0 ; \quad \eta_{\alpha} \circ f=0 ; \quad f^{2}=-I+\sum_{\alpha} \xi_{\alpha} \otimes \eta_{\alpha} ; \\
g(X, Y)=g(f X, f Y)+\Phi(X, Y),
\end{gathered}
$$

for any $X, Y \in T(N), \alpha=1 \ldots, s$, , where $\Phi(X, Y)=\sum_{\alpha} \eta_{\alpha}(X) \eta_{\alpha}(Y)$.

(ii) The $f$-structure $f$ is normal, that is,

$$
[f, f]+2 \sum_{\alpha} \xi \alpha \otimes \mathrm{d} \eta_{\alpha}=0
$$

where $[f, f]$ is the Nijenhuis torsion of $f$.

(iii) $\eta_{1} \wedge \ldots \wedge \eta_{s} \wedge\left(d \eta_{\alpha}\right)^{n} \neq 0$ and $d \eta_{1}=\ldots=d \eta_{s}=F$, for any $\alpha$, where $F$ is the fundamental 2 -form defined by $F(X, Y)=g(X, f Y), X, Y \in T(N)$. 
In the case $s=1$, an $S$-manifold is a Sasakian manifold. For $s \geq 2$, examples of $S$-manifolds are given in [1], [2], [3], [5].

For the Riemannian connection $\widetilde{\nabla}$ of $g$ on an $S$-manifold $N^{2 n+s}$, the following were also proved in [1]:

$$
\begin{aligned}
\widetilde{\nabla}_{X} \xi_{\alpha} & =-f X, \quad X \in T(N), \alpha=1, \ldots, s, \\
\left(\widetilde{\nabla}_{X} f\right) Y & =\sum_{\alpha}\left[g(f X, f Y) \xi_{\alpha}+\eta_{\alpha}(Y) f^{2} X\right], \quad X, Y \in T(Y) .
\end{aligned}
$$

Let $\mathcal{L}$ denote the distribution determined by $-f^{2}$ and $\mathcal{M}$ the complementary distribution. $\mathcal{M}$ is determined by $f^{2}+I$ and spanned by $\xi_{1}, \ldots, \xi_{s}$. If $X \in \mathcal{L}$, then $\eta_{\alpha}(X)=0$, for any $\alpha$, and if $X \in \mathcal{M}$, then $f X=0$.

A plane section $\pi$ is called an invariant $f$-section if it is determined by a vector $X \in \mathcal{L}(p), p \in N^{2 n+s}$, such that $\{X, f X\}$ is an orthonormal pair spanning the section. The sectional curvature $K(X, f X)$, denoted by $H(X)$, is called an invariant $f$-sectional curvature. If $N^{2 n+s}$ is an $S$-manifold of constant invariant $f$-sectional curvature $k$, then its curvature tensor has the form $([6])$

$$
\begin{aligned}
& \widetilde{R}(X, Y, Z, W)=\sum_{\alpha, \beta}\left\{g(f X, f W) \eta_{\alpha}(Y) \eta_{\beta}(Z)\right. \\
& \quad-g(f X, f Z) \eta_{\alpha}(Y) \eta_{\beta}(W)+g(f Y, f Z) \eta_{\alpha}(X) \eta_{\beta}(W) \\
& \left.\quad-g(f Y, f W) \eta_{\alpha}(X) \eta_{\beta}(Z)\right\}+\frac{1}{4}(k+3 s)\{g(X, W) g(f Y, f Z) \\
& \quad-g(X, Z) g(f Y, f W)+g(f Y, f W) \Phi(X, Z) \\
& \quad-g(f Y, f Z) \Phi(X, W)\}+\frac{1}{4}(k-s)\{F(X, W) F(Y, Z) \\
& \quad-F(X, Z) F(Y, W)-2 F(X, Y) F(Z, W)\}, \quad X, Y, Z, W \in T(N) .
\end{aligned}
$$

Then the $S$-manifold will be denoted by $N^{2 n+s}(k)$.

Now, let $M^{m}$ be an $m$-dimensional submanifold immersed in an $S$ manifold $N^{2 n+s}$. For any $X \in T(M)$, we write

$$
f X=T X+N X,
$$

where $T X$ is the tangential component of $f X$ and $N X$ is the normal component of $f X$. Then $T$ is an endomorphism of the tangent bundle and $N$ is a normal-bundle valued 1-form on the tangent bundle.

The submanifold $M^{m}$ is said to be invariant if all $\xi_{\alpha}(\alpha=1, \ldots, s)$ are always tangent to $M^{m}$ and $N$ is identically zero, i.e., $f X \in T(M)$, for any $X \in T(M)$. It is easy to show that an invariant submanifold of an $S$-manifold is an $S$-manifold too and so $m=2 p+s$. On the other hand, $M^{m}$ is said to be an anti-invariant submanifold if $T$ is identically zero, i.e., $f X \in T(M)^{\perp}$, for any $X \in T(M)$.

From now on, we suppose that $M^{m}$ is tangent to the structure vector 
fields (then $m \geq s$ ). From (2.2) and (2.5), we easily get

(2.6) $\nabla_{X} \xi_{\alpha}=-T X ; \quad \sigma\left(X, \xi_{\alpha}\right)=-N X, \quad X \in T(M), \quad \alpha=1, \ldots, s$.

Lemma 2.1. Let $M^{2 p+s}$ be an invariant submanifold of an $S$-manifold $N^{2 n+s}$. Then, for any $X, Y \in T(M)$,

$$
\sigma(X, f Y)=f \sigma(X, Y)=\sigma(f X, Y) .
$$

Proof. By using (2.3) and the Gauss-Weingarten formulas, we obtain

$$
\begin{aligned}
& \sigma(X, f Y)=\widetilde{\nabla}_{X} f Y-\nabla_{X} f Y=\left(\widetilde{\nabla}_{X} f\right) Y+f \widetilde{\nabla}_{X} Y-\nabla_{X} f Y \\
& =\sum_{\alpha}\left\{g(f X, f Y) \xi_{\alpha}+\eta_{\alpha}(Y) f^{2} x\right\}+f \nabla_{X} Y+f \sigma(X, Y)-\nabla_{X} f Y .
\end{aligned}
$$

Now, since $M^{2 p+s}$ is an invariant submanifold, comparing the normal parts yields (2.7).

Proposition 2.2. Let $M^{2 p+s}$ be an invariant submanifold of an S-manifold $N^{2 n+s}$. If $H$ denotes the invariant $f$-sectional curvature of $M^{2 p+s}$ and $\widetilde{H}$ denotes the invariant $f$-sectional curvature of $N^{2 n+s}$, then $H \leq \widetilde{H}$ and equality holds if and only if $M^{2 p+s}$ is totally geodesic.

Pro of. By using the Gauss equation (1.2) and (2.7), we easily prove

$$
R(X, f X, f X, X)=\widetilde{R}(X, f X, f X, X)-2\|\sigma(X, X)\|^{2},
$$

for any $X \in T(M)$. Then the first assertion is immediate from (2.8). Now, if $M^{2 p+s}$ is totally geodesic, then $\sigma(X, X)=0$, for any $X \in T(M)$, and $H=\widetilde{H}$. Conversely, if $H=\widetilde{H}$, then $\sigma(X, X)=0$, for any unit vector field $X \in T(M)$. Now, since $\sigma$ is symmetric, the proof is complete.

Proposition 2.3. If the second fundamental form $\sigma$ on an invariant submanifold $M^{2 p+s}$ of an $S$-manifold $N^{2 n+s}$ is parallel, then $M^{2 p+s}$ is totally geodesic.

Pro of. From (2.6), we have $\sigma\left(X, \xi_{\alpha}\right)=0$, for any $X \in T(M)$ and any $\alpha$, because $M^{2 p+s}$ is an invariant submanifold. Now, since $M^{2 p+s}$ is an $S$-manifold too, from (1.4) and (2.2) we get

$$
0=\left(\nabla_{X}^{\prime} \sigma\right)\left(Y, \xi_{\alpha}\right)=f \sigma(X, Y),
$$

for any $X, Y \in T(M)$, so that $\sigma \equiv 0$ and $M^{2 p+s}$ is totally geodesic.

Proposition 2.4. Let $M^{m+s}$ be a submanifold tangent to the structure vector fields of an $S$-manifold $N^{2 n+s}(k) \quad(k \neq s)$. Then $(\widetilde{R}(X, Y) Z)^{\perp}=0$, for any $X, Y, Z \in T(M)$, if and only if $M^{m+s}$ is invariant or anti-invariant.

Proof. If $M^{m+s}$ is invariant or anti-invariant, from (2.4) we easily have $(\widetilde{R}(X, Y) Z)^{\perp}=0, X, Y, Z \in T(M)$. Conversely, if $(\widetilde{R}(X, Y) Z)^{\perp}=0$, from 
(2.4) we get

$$
\begin{aligned}
0=\widetilde{R}(X, Y, Z, V)= & \frac{1}{4}(k-s)\{F(X, V) F(Y, Z)-F(X, Z) F(Y, V) \\
& -2 F(X, Y) F(Z, V)\}, \quad V \in T(M)^{\perp} .
\end{aligned}
$$

Putting $X=Z$, we obtain $0=g(Y, f X) g(X, f V)$, for any $X, Y \in T(M)$ and $V \in T(M)^{\perp}$. Then $M^{m+s}$ is an invariant or anti-invariant submanifold.

3. Proof of Theorem 1. Let $M^{m+s}$ be a submanifold of $N^{2 n+s}(k)$ $(k \neq s)$, tangent to the structure vector fields and with parallel second fundamental form. Then the Codazzi equation (1.3) reduces to $(\widetilde{R}(X, Y) Z)^{\perp}=0$, for any $X, Y, Z \in T(M)$. So, from Proposition 2.4, we find that $M^{m+s}$ is invariant or anti-invariant. If $M^{m+s}$ is invariant, Propositions 2.2 and 2.3 prove (a).

Now, assume that $M^{m+s}$ is anti-invariant. Then the normal space $T_{p}(M)^{\perp}$, at any point $p \in M^{m+s}$, can be decomposed as

$$
T_{p}(M)^{\perp}=f T_{p}(M) \oplus \nu_{p}(M),
$$

where $\nu_{p}(M)$ is the orthogonal complement of $f T_{p}(M)$ in $T_{p}(M)^{\perp}$. Now, since $\sigma$ is parallel, from (2.6) it is easy to prove that

$$
D_{X} f Y=f \nabla_{X} Y, \quad X, Y \in T(M),
$$

that is, $f T(M)$ is parallel with respect to the normal connection. Moreover, by using the Gauss-Weingarten formulas and (2.3), we get, for any $X, Y \in$ $T(M)$,

$$
\begin{aligned}
A_{f Y} X=-\widetilde{\nabla}_{X} f Y+D_{X} f Y= & -\sum_{\alpha}\left\{g(f X, f Y) \xi_{\alpha}+\eta_{\alpha}(Y) f^{2} X\right\} \\
& -f \nabla_{X} Y-f \sigma(X, Y)+D_{X} f Y .
\end{aligned}
$$

Therefore, we have

$$
f A_{f Y} X-\sum_{\alpha} \eta_{\alpha}(Y) f X-\sigma(X, Y)=0 .
$$

So, for any $W \in \nu$, we obtain $g(\sigma(X, Y), W)=0$, and consequently

$$
A_{W}=0 \text {. }
$$

Since $f T(M)$ is of constant dimension on $M^{m+s}$ and taking account of (3.1) and (3.2), from the reduction theorem of Erbacher ([4]), there exists a totally geodesic invariant submanifold $\bar{M}^{2 m+s}(k)$ in $N^{2 n+s}(k)$, where $M^{m+s}$ is immersed in $\bar{M}^{2 m+s}(k)$ as an anti-invariant submanifold. This completes the proof.

4. Examples. Let $E^{2 n+s}$ be a euclidean space with cartesian coordinates $\left(x_{1}, \ldots, x_{n}, y_{1}, \ldots, y_{n}, z_{1}, \ldots, z_{s}\right)$. Then an $S$-structure on $E^{2 n+s}$ is 
defined by (cf. [5])

$$
\begin{aligned}
& \xi_{\alpha}=2 \partial / \partial z_{\alpha} \quad(\alpha=1, \ldots, s) \\
& \eta_{\alpha}=\frac{1}{2}\left(d z_{\alpha}-\sum_{i=1}^{n} y_{i} d x_{i}\right) \quad(\alpha=1, \ldots, s) \\
& f X=\sum_{i=1}^{n} Y^{i} \partial / \partial x_{i}-\sum_{i=1}^{n} X^{i} \partial / \partial y_{i}+\left(\sum_{i=1}^{n} Y^{i} y_{i}\right)\left(\sum_{\alpha} \partial / \partial z_{\alpha}\right) ; \\
& g=\sum_{\alpha} \eta_{\alpha} \otimes \eta_{\alpha}+\frac{1}{4} \sum_{i=1}^{n}\left(d x_{i} \otimes d x_{i}+d y_{i} \otimes d y_{i}\right)
\end{aligned}
$$

where $X=\sum_{i=i}^{n}\left(X^{i} \partial / \partial x_{i}+Y^{i} \partial / \partial y_{i}\right)+\sum_{\alpha} Z^{\alpha} \partial / \partial z_{\alpha}$.

With this structure, $E^{2 n+s}$ is an $S$-manifold of constant invariant $f$ sectional curvature $k=-3 s([5])$.

(1) We consider the following natural imbedding of $E^{n+s}$ into $E^{2 n+s}(-3 s)$ :

$$
\left(x_{1}, \ldots, x_{n}, z_{1}, \ldots, z_{s}\right) \mapsto\left(x_{1}, \ldots, x_{n}, 0, \ldots, 0, z_{1}, \ldots, z_{s}\right) .
$$

A frame field for tangent vector fields in $E^{n+s}$ is given by $\left\{X_{1}, \ldots, X_{n}\right.$, $\left.\xi_{1}, \ldots, \xi_{s}\right\}$, where $X_{i}=\partial / \partial x_{i}(i=1, \ldots, n)$. Then it is easy to check that $E^{n+s}$ is an anti-invariant submanifold of $E^{2 n+s}(-3 s)$. Moreover, we have $\sigma\left(X_{i}, X_{j}\right)=(s / 2)\left(y_{j} f X_{i}+y_{i} f X j\right)$ and, from $(2.6), \sigma\left(X_{i}, \xi_{\alpha}\right)=-f X_{i}$, $\sigma\left(\xi_{\alpha}, \xi_{\beta}\right)=0,(i, j=1, \ldots, n, \alpha, \beta=1, \ldots, s)$. Thus, the second fundamental form of $E^{n+s}$ in $E^{2 n+s}(-3 s)$ is parallel.

On the other hand, $E^{2 m+s}(-3 s)$ is a totally geodesic and invariant submanifold of $E^{2 n+s}(-3 s) \quad(m<n)$.

(2) Let $S^{1}=\{z \in \mathbb{C}:|z|=1\}$, and put

$$
M^{n+s}=S^{1} \times E^{n-1} \times E^{s} .
$$

Then consider an imbedding of $M^{n+s}$ into $E^{2 n+s}(-3 s)$ given by

$$
\left(\cos u, x_{2}, \ldots, x_{n}, \sin u, 0, \ldots, 0, z_{1}, \ldots, z_{s}\right) .
$$

A frame field for tangent vector fields in $M^{n+s}$ is given by $\left\{X_{1}, \ldots, X_{n}\right.$, $\left.\xi_{1}, \ldots, \xi_{s}\right\}$, where

$$
\begin{aligned}
& X_{1}=-\sin u \partial / \partial x_{1}+\cos u \partial / \partial y_{1} ; \\
& X_{i}=\partial / \partial x_{i} \quad(i=2, \ldots, n) .
\end{aligned}
$$

Thus, $M^{n+s}$ is an anti-invariant submanifold of $E^{2 n+s}(-3 s)$. Moreover, the second fundamental form of $M^{n+s}$ in $E^{2 n+s}(-3 s)$ is given by

$$
\begin{aligned}
\sigma\left(X_{1}, X_{1}\right) & =-\left(1+s y_{1}^{2}\right) f X_{1} \\
\sigma\left(X_{1}, X_{i}\right) & =(s / 2)\left(y_{i} f X_{1}-y_{1}^{2} f X_{i}\right) \quad(i=2, \ldots, n)
\end{aligned}
$$




$$
\begin{aligned}
\sigma\left(X_{i}, X_{j}\right) & =(s / 2)\left(y_{i} f X_{j}+y_{j} f X_{i}\right) \quad(i, j=2, \ldots, n) ; \\
\sigma\left(X_{i}, \xi_{\alpha}\right) & =-f X_{i} \quad(i=1, \ldots, n, \alpha=1, \ldots, s) ; \\
\sigma\left(\xi_{\alpha}, \xi_{\beta}\right) & =0 \quad(\alpha, \beta=1, \ldots, s) .
\end{aligned}
$$

Then the second fundamental form of $M^{n+s}$ is parallel.

(3) Let $S^{2 n+1}$ be the $(2 n+1)$-dimensional unit sphere with the standard Sasakian structure. Then $S^{2 n+1}$ is of constant invariant $f$-sectional curvature $k=1$ (cf. [7]). If we consider the Clifford hypersurface $M_{p, q}$ defined by

$$
M_{p, q}=S^{p}(\sqrt{(p / 2 n)}) \times S^{q}(\sqrt{(q / 2 n)}), \quad p+q=2 n,
$$

then $M_{p, q}$ is tangent to the structure vector field $\xi$, has parallel second fundamental form, but is neither an invariant nor an anti-invariant submanifold of $S^{2 n+1}$.

Therefore, the assumption in Theorem 1 on the invariant $f$-sectional curvature $k \neq s$ of the ambient $S$-manifold is essential.

\section{References}

[1] D. E. Blair, Geometry of manifolds with structural group $U(n) \times O(s)$, J. Differential Geom. 4 (1970), 155-167.

[2] - On a generalization of the Hopf fibration, An. Stiinţ. Univ. "Al. I. Cuza" Iaşi 17 (1) (1971), 171-177.

[3] D. E. Blair, G. D. Ludden and K. Yano, Differential geometric structures on principal toroidal bundles, Trans. Amer. Math. Soc. 181 (1973), 175-184.

[4] J. Erbacher, Reduction of the codimension of an isometric immersion, J. Differential Geom. 5 (1971), 333-340.

[5] I. Hasegawa, Y. Okuy ma and T. Abe, On p-th Sasakian manifolds, J. Hokkaido Univ. Ed. Sect. II A 37 (1) (1986), 1-16.

[6] M. Kobayashi and S. Tsuchiya, Invariant submanifolds of an $f$-manifold with complemented frames, Kôdai Math. Sem. Rep. 24 (1972), 430-450.

[7] S. Tanno, Sasakian manifolds with constant $\psi$-holomorphic sectional curvature, Tôhoku Math. J. 21 (1969), 501-507.

[8] K. Yano, On a structure defined by a tensor field $f$ of type $(1,1)$ satisfying $f^{3}+f=0$, Tensor 14 (1963), 99-109.

DEPARTAMENTO DE ALGEBRA, COMPUTACIÓN, GEOMETRÍA Y TOPOLOGÍA FACULTAD DE MATEMÁTICAS

UNIVERSIDAD DE SEVILLA

APARTADO DE CORREOS 1.160

41080 SEVILLA, SPAIN 